\title{
Stereotactic laser ablation of amygdala and hippocampus using a Leksell stereotactic frame
}

\author{
Ahmed J. Awad, MD, ${ }^{1,2}$ Ha S. Nguyen, MD, ${ }^{1}$ Elsa Arocho-Quinones, MD, ${ }^{1}$ Ninh Doan, MD, PhD, ${ }^{1}$ \\ Wade Mueller, MD, ${ }^{1}$ and Sean M. Lew, MD ${ }^{1}$ \\ 1Department of Neurosurgery, Medical College of Wisconsin, Milwaukee, Wisconsin; and \\ ${ }^{2}$ Faculty of Medicine and Health Sciences, An-Najah National University, Palestine \\ Approximately one-third of patients with epilepsy are resistant to medical therapy, particularly in those with mesial \\ temporal lobe epilepsy. While there are several surgical modalities, efforts have been focused on developing safer and \\ minimally invasive techniques. In this video, the authors present the case of a 45-year-old woman with a 2-year history of \\ refractory left mesial temporal lobe epilepsy who underwent MRI-guided laser ablation of amygdala and hippocampus. \\ There were no perioperative complications.
}

The video can be found here: https://youtu.be/XFHt2jTdE_4.

KEYWORDS laser; ablation; thermal; stereotactic; Visualase; sclerosis; epilepsy; seizures 